\title{
REDUCTION FOR LOCALLY CONFORMAL SYMPLECTIC MANIFOLDS
}

\author{
Stefan Haller and Tomasz Rybicki
}

\begin{abstract}
It is shown how one can do symplectic reduction for locally conformal symplectic manifolds, especially with an action of a Lie group. This generalizes well known procedures for symplectic manifolds to the slightly larger class of locally conformal symplectic manifolds. The whole setting is very conformally invariant.
\end{abstract}

\section{INTRODUCTION}

The geometry of locally conformal symplectic (l.c.s. for short) structures generalizes the symplectic geometry. Such structures occur naturally in the theory of Jacobi manifolds, as any even-dimensional leaf of the distinguished foliation of a Jacobi manifold is l.c.s., cf.[2]. In particular, any transitive even-dimensional Jacobi manifold is actually an l.c.s. manifold. The l.c.s. manifolds may be regarded as natural phase spaces in the Hamiltonian mechanics ([8]). For instance, in [9] the symmetry of the Lyapunov spectrum in locally conformal Hamiltonian systems is studied. The authors of [9] present several examples and show that Gaussian isokinetic dynamics, Nosé-Hoovers dynamics and other systems can be viewed as locally conformal Hamiltonian systems.

The aim of this note is to show that, in regular cases, the symplectic reduction is possible for l.c.s. manifolds. The most abstract reduction principle states that every coisotropic submanifold of a symplectic manifold is foliated by isotropic leaves and if the leaf space is a manifold it carries again a symplectic structure. In the paper of Marsden and Weinstein [7] it has been formalized the fact that if an $n$ dimensional symmetry group acts on a Hamiltonian system then the number of degrees of freedom can be reduced by $n$, and the dimension of the phase space is reduced by $2 n$ (the position and the momentum coordinates). We show that this is still true for l.c.s. manifolds. Moreover the reduction process preserves the conformal equivalence class of an l.c.s. structure. An open problem is how to treat singular reductions (cf.[1]) in the l.c.s. case.

\section{Locally Conformal Symplectic Manifolds}

For a closed 1-form $\omega \in \Omega^{1}(M)$ and a vector field $X \in \mathfrak{X}(M)$ we define $d^{\omega}$ : $\Omega^{*}(M) \rightarrow \Omega^{*+1}(M)$ by $d^{\omega} \alpha:=d \alpha+\omega \wedge \alpha$, and $L_{X}^{\omega}: \Omega^{*}(M) \rightarrow \Omega^{*}(M)$ by $L_{X}^{\omega} \alpha:=$ $L_{X} \alpha+\omega(X) \alpha$. The well known formulas $d^{\omega} d^{\omega}=0, L_{X}^{\omega} L_{Y}^{\omega}-L_{Y}^{\omega} L_{X}^{\omega}=L_{[X, Y]}^{\omega}$,

1991 Mathematics Subject Classification. 53D20.

Key words and phrases. locally conformal symplectic manifolds, symplectic reduction.

Typeset by $\mathcal{A M}_{\mathcal{M}}-\mathrm{T}_{\mathrm{E}} \mathrm{X}$ 
$L_{X}^{\omega} d^{\omega}-d^{\omega} L_{X}^{\omega}=0, L_{X}^{\omega} i_{Y}-i_{Y} L_{X}^{\omega}=i_{[X, Y]}$ and $d^{\omega} i_{X}+i_{X} d^{\omega}=L_{X}^{\omega}$ remain true. Moreover one has the derivation like formulas

$$
\begin{aligned}
& d^{\omega_{1}+\omega_{2}}(\alpha \wedge \beta)=d^{\omega_{1}} \alpha \wedge \beta+(-1)^{|\alpha|} \alpha \wedge d^{\omega_{2}} \beta \text { and } \\
& L_{X}^{\omega_{1}+\omega_{2}}(\alpha \wedge \beta)=L_{X}^{\omega_{1}} \alpha \wedge \beta+\alpha \wedge L_{X}^{\omega_{2}} \beta .
\end{aligned}
$$

A locally conformal symplectic manifold is a triple $(M, \Omega, \omega)$, where $\Omega \in \Omega^{2}(M)$ is non-degenerate, $\omega \in \Omega^{1}(M)$ is closed and $d^{\omega} \Omega=0$. If $\operatorname{dim}(M)>2$ then $\omega$ is uniquely determined by $\Omega$. Two l.c.s. structures $(\Omega, \omega)$ and $\left(\Omega^{\prime}, \omega^{\prime}\right)$ are called conformally equivalent (we will write $(\Omega, \omega) \sim\left(\Omega^{\prime}, \omega^{\prime}\right)$ ) if there exists a positive function $a$ such that $\Omega^{\prime}=\frac{1}{a} \Omega$ and $\omega^{\prime}=\omega+\frac{d a}{a}=\omega+d \ln a$. Notice that, if $\operatorname{dim}(M)>2$, the second equation is a consequence of the first.

Suppose we have a manifold $M$, an open covering $\mathcal{U}$ of $M$ and on every $U \in \mathcal{U}$ an l.c.s. structure $\left(\Omega_{U}, \omega_{U}\right)$, such that $\left.\left.\left(\Omega_{U}, \omega_{U}\right)\right|_{U \cap V} \sim\left(\Omega_{V}, \omega_{V}\right)\right|_{U \cap V}$ for all $U, V \in \mathcal{U}$. Then there exists, up to conformal equivalence, a unique l.c.s. structure $(\Omega, \omega)$ on $M$ with $\left.(\Omega, \omega)\right|_{U} \sim\left(\Omega_{U}, \omega_{U}\right)$ for all $U \in \mathcal{U}$. It can be constructed as follows. For every pair $U, V \in \mathcal{U}$ there exists a function $a_{U V}: U \cap V \rightarrow \mathbb{R}^{+}$, such that $\left.\Omega_{U}\right|_{U \cap V}=$ $\left.\frac{1}{a_{U V}} \Omega_{V}\right|_{U \cap V}$ and $\left.\omega_{U}\right|_{U \cap V}=\left.\omega_{V}\right|_{U \cap V}+\frac{d a_{U V}}{a_{U V}}$. Obviously we have $a_{U V} a_{V W}=a_{U W}$ on $U \cap V \cap W$. In other words $\left\{a_{U V}\right\}$ is a 1-cocycle in the Cech-cohomology of the sheaf $C^{\infty}\left(\cdot, \mathbb{R}^{+}\right)$. Since this sheaf is fine, and hence acyclic, every cocycle is a coboundary, that is there exist functions $b_{U}: U \rightarrow \mathbb{R}^{+}$with $a_{U V}=\frac{b_{V}}{b_{U}}$ on $U \cap V$. Now one defines $\left.\Omega\right|_{U}:=\frac{1}{b_{U}} \Omega_{U}$ and $\left.\omega\right|_{U}:=\omega_{U}+\frac{d b_{U}}{b_{U}}([5])$.

By $\operatorname{Diff}^{\infty}(M, \Omega, \omega)$ we denote the group of all diffeomorphisms $M$, which preserve $(\Omega, \omega)$ up to conformal equivalence. The corresponding Lie algebra of vector fields is

$\mathfrak{X}(M, \Omega, \omega)=\left\{X \in \mathfrak{X}(M): L_{X}^{\omega} \Omega=c_{X} \Omega\right.$, For some locally constant $\left.c_{X}\right\}$,

cf.[4].

$C^{\infty}(M, \mathbb{R})$ becomes a Lie algebra with the following bracket

$$
\left\{f_{1}, f_{2}\right\}:=\Omega\left(\sharp d^{\omega} f_{1}, \sharp d^{\omega} f_{2}\right)=L_{\sharp d^{\omega} f_{2}}^{\omega} f_{1}=-L_{\sharp d^{\omega} f_{1}}^{\omega} f_{2} .
$$

Here $\sharp$ denotes the inverse of $b$, where $b: T M \cong T^{*} M$ given by $X \mapsto i_{X} \Omega$ is a canonical vector bundle isomorphism since $\Omega$ is non-degenerate.

We have $\sharp d^{\omega}\left\{f_{1}, f_{2}\right\}=-\left[\sharp d^{\omega} f_{1}, \sharp d^{\omega} f_{2}\right]$. So

$$
\operatorname{Ham}(M, \Omega, \omega):=\left\{\sharp d^{\omega} f: f \in C^{\infty}(M, \mathbb{R})\right\}
$$

is a subalgebra of $\mathfrak{X}(M, \Omega, \omega)$, and

$$
H_{d^{\omega}}^{0}(M) \hookrightarrow C^{\infty}(M, \mathbb{R}) \stackrel{\sharp d^{\omega}}{\longrightarrow} \operatorname{Ham}(M, \Omega, \omega)
$$

is, up to a sign, a central extension of Lie algebras. Here $H_{d^{\omega}}^{*}(M)$ denotes the cohomology with respect to the differential $d^{\omega}$, especially

$$
H_{d^{\omega}}^{0}(M)=\left\{f \in C^{\infty}(M, \mathbb{R}): d^{\omega} f=0\right\} .
$$

Note that if $\left(\Omega^{\prime}, \omega^{\prime}\right) \sim(\Omega, \omega)$, i.e. $\Omega^{\prime}=\frac{1}{a} \Omega$ and $\omega^{\prime}=\omega+\frac{d a}{a}$, then $\{f, g\}^{\prime}=$ $\frac{1}{a}\{a f, a g\}$. Moreover one has $\{f g, h\}=f\{g, h\}+\{f, h\} g-f g L_{\sharp \omega} h$. 


\section{REDUCTION FOR L.C.S. STRUCTURES}

We begin with the following general definition of reduction essentially due to Marsden and Ratiu [6].

Definition. Let $(M, \Omega, \omega)$ be an l.c.s. manifold, and let $\{., .\}_{M}$ be the corresponding bracket on $C^{\infty}(M, \mathbb{R})$. A pair $(L, E)$, where $i: L \subset M$ is a submanifold and $E$ is a vector subbundle of $\left.T M\right|_{L}$, is said to be a reductive structure if the following is fulfilled:

(1) $E \cap T L$ is tangent to a foliation of $L$ whose leaves are the fibers of a submersion $\pi: L \rightarrow P_{L}$

(2) $i^{*} \omega(X)=0$ for any $X$ tangent to the fibers of $\pi$; and

(3) for any $u, v \in C^{\infty}(M, \mathbb{R})$ such that $d u, d v$ vanish on $E d^{\omega}\{u, v\}_{M}$ vanishes on $E$ as well.

Furthermore, $(M, L, E)$ is called a reducible triple if there is an l.c.s. structure $(\bar{\Omega}, \bar{\omega})$ on $P_{L}$ with the Poisson bracket $\{., .\}_{P_{L}}$ such that for any local $C^{\infty}$ functions $f, g$ on $P_{L}$, and any local extensions $u, v$ of $f \circ \pi, g \circ \pi$ respectively, such that both $d^{\omega} u$ and $d^{\omega} v$ vanish on $E$ one has the relation

$$
\{u, v\}_{M} \circ i=\{f, g\}_{P_{L}} \circ \pi
$$

$\left(P_{L}, \bar{\Omega}, \bar{\omega}\right)$ is then a reduced l.c.s. manifold.

A standard reductive structure arise in the following situation. Let $(M, \Omega, \omega)$ be an l.c.s. manifold such that for a submanifold $i: L \subset M \operatorname{rank}\left(i^{*} \Omega\right)$ is constant. Then it is apparent that for the $\Omega$-orthogonal space $T L^{\perp}$ of $T L, T L^{\perp} \cap T L$ is integrable. Hence if the resulting foliation is given by a submersion $\pi: L \rightarrow P_{L}$ and $i^{*} \omega$ vanishes on $T L^{\perp} \cap T L$ then $\left(L, T L^{\perp} \cap T L\right)$ is a reductive structure. By Proposition 1 below we see that $\left(M, L, T L^{\perp} \cap T L\right)$ is a reducible triple as well since $\sharp \operatorname{Ann}\left(T L^{\perp}\right)=\left(T L^{\perp}\right)^{\perp}=T L$.

As in the symplectic and Poisson case [6] we have

Proposition 1. Let $(M, \Omega, \omega)$ be an l.c.s. manifold and suppose $(L, E)$ is a reductive structure. Then $(M, L, E)$ is a reducible triple if and only if

$$
\sharp(\text { Ann } E) \subset T L+E \text {. }
$$

Moreover, if $(M, \Omega, \omega) \sim\left(M, \Omega^{\prime}, \omega^{\prime}\right)$ then $\left(P_{L}, \bar{\Omega}, \bar{\omega}\right) \sim\left(P_{L}, \bar{\Omega}^{\prime}, \bar{\omega}^{\prime}\right)$, i.e. the conformal equivalence relation is preserved.

Proof. Assume that $\left(P_{L}, \bar{\Omega}, \bar{\omega}\right)$ is a reduced l.c.s. of $(M, \Omega, \omega)$. Let $x \in L, \alpha \in$ Ann $E_{x}$, and $\beta \in \operatorname{Ann}\left(E_{x}+T_{x} L\right)=\left(\operatorname{Ann} E_{x}\right) \cap\left(\operatorname{Ann} T_{x} L\right)$. By (1) there is $u \in$ $C^{\infty}(M, \mathbb{R})$ such $d_{x} u=\alpha$ and $\left.d u\right|_{E}=0$. Now due to $(2) d_{x}^{\omega} u=\alpha$ and $d^{\omega} u$ vanishes on $E$. Likewise, we choose $v \in C^{\infty}(M, \mathbb{R})$ vanishing on $L$ with $d_{x} v=\beta$ and $\left.d v\right|_{E}=0$. Hence in view of (2) $d_{x}^{\omega} v=\beta$ and $d^{\omega} v$ vanishes on $E$. Consequently, by the definition of reducible triple, we have

$$
\begin{aligned}
\beta(\sharp \alpha) & =\left(d_{x}^{\omega} v\right)\left(\sharp d_{x}^{\omega} u\right)=\left(d_{x} v\right)\left(\sharp d_{x}^{\omega} u\right)=\left(L_{\sharp d_{x}^{\omega} u} v\right)(x) \\
& =\{v, u\}_{M}(x)=\{0, f\}(\pi(x))_{P_{L}}=0,
\end{aligned}
$$

where $f \in C^{\infty}\left(P_{L}, \mathbb{R}\right)$ such that $u$ extends $f \circ \pi$. This gives the relation $(*)$. 
Conversely, suppose $(*)$ is fulfilled and let $f, g \in C^{\infty}\left(P_{L}, \mathbb{R}\right)$. For fixed $x \in L$ choose extensions $u, v \in C^{\infty}(M, \mathbb{R})$ of $f \circ \pi, g \circ \pi$ resp. such that $u(x)=v(x)=0$ and $\left.d u\right|_{E}=\left.d v\right|_{E}=0$. This yields $\left.d\{u, v\}_{M}\right|_{E}=\left.d^{\omega}\{u, v\}_{M}\right|_{E}=0$ and, consequently, $\left.\{u, v\}_{M}\right|_{L}$ is constant along fibers. We let $\{f, g\}_{P_{L}}(\pi(x)):=\{u, v\}_{M}(x)$ which will ensure that $(M, L, E\}$ is a reducible triple. The only thing to check is the independence of the choice of $u$ and $v$. If $\tilde{u}$ also extends $f \circ \pi$ with $\tilde{u}(x)=0$ and $\left.d^{\omega} \tilde{u}\right|_{E}=0$, we get $\left.(u-\tilde{u})\right|_{L}=0$. Therefore $d u=d \tilde{u}$ on $E+T L$ which, in view of (*) implies

$$
\{u, v\}_{M}(x)=\left(d_{x}^{\omega} u\right)\left(\sharp d_{x}^{\omega} v\right)=\left(d_{x}^{\omega} \tilde{u}\right)\left(\sharp d_{x}^{\omega} v\right)=\{\tilde{u}, v\}_{M}(x) .
$$

By the antisymmetry the definition is also independent of the choice of $v$.

The second assertion is obvious.

\section{The Moment Mapping}

Let $G$ be a finite dimensional Lie group acting from the left on $M$. We will write $l_{g}: M \rightarrow M$ for the action of the element $g \in G$. For the fundamental vector fields $\zeta_{X}$ we have $\zeta_{[X, Y]}=-\left[\zeta_{X}, \zeta_{Y}\right]$ and $\zeta_{\operatorname{Ad}_{g^{-1}} X}=l_{g}^{*} \zeta_{X}$. In the sequel we will always assume that $G$ acts symplectically, i.e. $l_{g} \in \operatorname{Diff}^{\infty}(M, \Omega, \omega)$ for all $g \in G$. If $G$ is connected this is equivalent to $\zeta_{X} \in \mathfrak{X}(M, \Omega, \omega)$ for all $X \in \mathfrak{g}$, where $\mathfrak{g}$ is the Lie algebra of $G$. Then for every $g \in G$ there exists a positive function $a_{g}$ on $M$, such that $l_{g}^{*} \Omega=\frac{1}{a_{g}} \Omega$ and $l_{g}^{*} \omega=\omega+\frac{d a_{g}}{a_{g}}$. Obviously we have $a_{h g}=a_{g}\left(l_{g}^{*} a_{h}\right)$. So we have a right $G$-action on $C^{\infty}(M, \mathbb{R})$ given by $g \cdot f:=a_{g}\left(l_{g}^{*} f\right)$. If one differentiates $l_{g}^{*} \Omega=\frac{1}{a_{g}} \Omega$ with respect to $X \in \mathfrak{g}$, one obtains $\left.\frac{\partial}{\partial t}\right|_{0} a_{\exp (t X)}=$ $\omega\left(\zeta_{X}\right)-c_{X}$. So the corresponding $\mathfrak{g}$-action on $C^{\infty}(M, \mathbb{R})$ is $X \cdot f=L_{\zeta_{X}}^{\omega} f-c_{X} f$. Moreover $\sharp d^{\omega}: C^{\infty}(M, \mathbb{R}) \rightarrow \operatorname{Ham}(M, \Omega, \omega)$ is equivariant, i.e. $\sharp d^{\omega}\left(a_{g} l_{g}^{*} f\right)=l_{g}^{*} \sharp d^{\omega} f$ and $\sharp d^{\omega}\left(L_{\zeta_{X}}^{\omega} f-c_{X} f\right)=\left[\zeta_{X}, \sharp d^{\omega} f\right]$.

Proposition 2. Let $G$ be a connected Lie group, and suppose the $\mathfrak{g}$-action is Hamiltonian, i.e. $\zeta_{X} \in \operatorname{Ham}(M, \Omega, \omega)$ for all $X \in \mathfrak{g}$. Let $\hat{\psi}: \mathfrak{g} \rightarrow C^{\infty}(M, \mathbb{R})$ be a linear lift of $\zeta$, that is $\sharp d^{\omega} \hat{\psi}(X)=\zeta_{X}$, for all $X \in \mathfrak{g}$. Moreover let $\psi: M \rightarrow \mathfrak{g}^{*}$ be defined by $\langle\psi(x), X\rangle=\hat{\psi}(X)(x)$, where $X \in \mathfrak{g}$ and $x \in M$. Then the following conditions

(1) $\hat{\psi}$ is a homomorphism of Lie algebras,

(2) $\hat{\psi}$ is equivariant, i.e. $\hat{\psi}\left(\operatorname{Ad}_{g^{-1}} X\right)=a_{g} l_{g}^{*} \hat{\psi}(X)$, for all $g \in G, X \in \mathfrak{g}$,

(3) $\hat{\psi}$ is infinitesimal invariant, i.e. $\hat{\psi}\left(-\operatorname{ad}_{Y} X\right)=L_{\zeta_{Y}}^{\omega} \hat{\psi}(X)$, for all $X, Y \in \mathfrak{g}$,

(4) $\operatorname{Ad}_{g^{-1}}^{*} \psi=a_{g} l_{g}^{*} \psi$, for all $g \in G$, and

(5) $-\operatorname{ad}_{Y}^{*} \psi=T \psi \cdot \zeta_{Y}+\omega\left(\zeta_{Y}\right) \psi$, for all $Y \in \mathfrak{g}$

are equivalent.

Proof. We have $\hat{\psi}\left(-\operatorname{ad}_{Y} X\right)=\hat{\psi}([X, Y])$ and

$$
L_{\zeta_{Y}}^{\omega} \hat{\psi}(X)=L_{\sharp d^{\omega} \hat{\psi}(Y)}^{\omega} \hat{\psi}(X)=\{\hat{\psi}(X), \hat{\psi}(Y)\} \text {. }
$$

So (1) is equivalent to (3), and since $G$ is connected (3) is equivalent to (2). Notice that since the action is Hamiltonian we have $c_{X}=0$ and so (3) is really the infinitesimal version of (2). Moreover (4) is obviously equivalent to (5). Next we 
have $\hat{\psi}\left(-\operatorname{ad}_{Y} X\right)(x)=\left\langle-\operatorname{ad}_{Y}^{*} \psi(x), X\right\rangle$, and

$$
\begin{aligned}
L_{\zeta_{Y}}^{\omega} \hat{\psi}(X)(x) & =L_{\zeta_{Y}} \hat{\psi}(X)(x)+\omega\left(\zeta_{Y}(x)\right) \hat{\psi}(X)(x) \\
& =L_{\zeta_{Y}}\langle\psi(x), X\rangle+\omega\left(\zeta_{Y}(x)\right)\langle\psi(x), X\rangle \\
& =\left\langle T_{x} \psi \cdot \zeta_{Y}(x), X\right\rangle+\left\langle\omega\left(\zeta_{Y}(x)\right) \psi(x), X\right\rangle,
\end{aligned}
$$

and thus (5) is equivalent to (3).

A mapping $\psi$ as in Proposition 2 is called a moment mapping, and it is called equivariant if the five equivalent conditions are satisfied.

Proposition 3. Let $\varphi: \mathfrak{g} \rightarrow \operatorname{Ham}(M, \Omega, \omega)$ be an anti homomorphism of Lie algebras. If $H^{2}\left(\mathfrak{g} ; H_{d^{\omega}}^{0}(M)\right)=0$, where $H_{d^{\omega}}^{0}(M)$ is considered as trivial $\mathfrak{g}$-module, then there exists a lift $\tilde{\varphi}: \mathfrak{g} \rightarrow C^{\infty}(M, \mathbb{R})$, i.e. $\tilde{\varphi}$ is a homomorphism of Lie algebras and $\sharp d^{\omega} \circ \tilde{\varphi}=\varphi$. Moreover, if there exists a lift, then the set of all lifts is parameterized by $H^{1}\left(\mathfrak{g} ; H_{d^{\omega}}^{0}(M)\right)$.

Proof. Let $\bar{\varphi}: \mathfrak{g} \rightarrow C^{\infty}(M, \mathbb{R})$ be a linear lift of $\varphi$ and set

$$
c(X, Y):=\bar{\varphi}([X, Y])-\{\bar{\varphi}(X), \bar{\varphi}(Y)\} \in H_{d^{\omega}}^{0}(M)
$$

Then one easily shows $\delta c=0$. Since $H^{2}\left(\mathfrak{g} ; H_{d^{\omega}}^{0}(M)\right)=0$ there exists $b$ with $\delta b=c$, i.e. $c(X, Y)=(\delta b)(X, Y)=-b([X, Y])$. Now we define $\tilde{\varphi}: \mathfrak{g} \rightarrow C^{\infty}(M, \mathbb{R})$ by $\tilde{\varphi}(X):=\bar{\varphi}(X)+b(X)$. Then one immediately sees that $\tilde{\varphi}$ is a homomorphism of Lie algebras and a lift of $\varphi$.

To see the second assertion let $\tilde{\varphi}$ denote a homomorphism of Lie algebras lifting $\varphi$. All other lifts are of the form $\tilde{\varphi}+b$ for some $b: \mathfrak{g} \rightarrow C^{\infty}(M, \mathbb{R})$. Since we have

$$
\{\tilde{\varphi}(X)+b(X), \tilde{\varphi}(Y)+b(Y)\}=\{\tilde{\varphi}(X), \tilde{\varphi}(Y)\}=\tilde{\varphi}([X, Y])
$$

we see that $\tilde{\varphi}+b$ is a homomorphism of Lie algebras iff $b([X, Y])=0$ for all $X, Y \in \mathfrak{g}$, or equivalently $\delta b=0$, i.e. $b \in H^{1}\left(\mathfrak{g} ; H_{d^{\omega}}^{0}(M)\right)$.

If $\mathfrak{g}$ is semi simple, then there always exists an equivariant moment mapping, since $H^{2}\left(\mathfrak{g} ; H_{d^{\omega}}^{0}(M)\right)=0$, by the second Whitehead lemma. Moreover it is unique since $H^{1}\left(\mathfrak{g} ; H_{d^{\omega}}^{0}(M)\right)=0$, by the first Whitehead lemma.

If $(M, \Omega, \omega)$ is an l.c.s. manifold which is not conformally equivalent to a symplectic manifold, i.e. $\omega$ is not exact, then there always exists a unique equivariant moment mapping. This is because $H_{d \omega}^{0}(M)=0$ for non-exact $\omega$.

Suppose the l.c.s. structure is exact, that is $\Omega=d^{\omega} \theta$, and the action preserves $\theta$, that is $L_{\zeta_{X}}^{\omega} \theta=c_{X} \theta$ for locally constant functions $c_{X}$. Notice that this immediately implies $L_{\zeta_{X}}^{\omega} \Omega=c_{X} \Omega$. Moreover $c_{X}=0$ iff the action is Hamiltonian, and in this situation $\hat{\psi}(X)=-i_{\zeta_{X}} \theta$ is an equivariant moment mapping.

\section{Lie Group Actions and Reduction}

Let $G$ be a finite dimensional Lie group acting symplectically on an l.c.s. manifold $(M, \Omega, \omega)$. Assume that we have an equivariant moment mapping $\psi$. The vector fields $Z \in \mathfrak{X}(M)$ which are $\Omega$-orthogonal to the orbits of $G$ span an involutive distribution on $M$. Indeed, since we have $\Omega\left(\zeta_{X}, Z\right)=i_{Z} d^{\omega} \hat{\psi}(X)=L_{Z}^{\omega} \hat{\psi}(X)$, this follows from $L_{\left[Z_{1}, Z_{2}\right]}^{\omega}=L_{Z_{1}}^{\omega} L_{Z_{2}}^{\omega}-L_{Z_{2}}^{\omega} L_{Z_{1}}^{\omega}$. Suppose $L$ is a maximal connected 
submanifold of $M$ which is tangent to this distribution, more precisely $T_{x} L=$ $\zeta_{\mathfrak{g}}(x)^{\perp}$ for all $x \in L$. Since we have $0=\left(L_{Z}^{\omega} \hat{\psi}(X)\right)(x)=\left\langle T_{x} \psi \cdot Z_{x}+\omega\left(Z_{x}\right) \psi(x), X\right\rangle$, wee see that $\psi(L) \subseteq \mathbb{R}^{+} \cdot \psi\left(x_{0}\right)$, for any $x_{0} \in L$. However $\psi$ need not be constant along $L$.

Let $\mathfrak{g}_{L}:=\left\{Y \in \mathfrak{g}: \operatorname{ad}_{Y}^{*} \psi\left(x_{0}\right)=0\right\}$, which does not depend on $x_{0} \in L$. By the equivariance of $\psi$, these are precisely those $Y$, for which $\zeta_{Y}$ is tangential to $L$. Since $L$ is maximal, the connected subgroup of $G$ corresponding to $\mathfrak{g}_{L}$ leaves $L$ invariant.

Theorem 1. Let $G$ be a finite dimensional Lie group acting symplectically on an l.c.s. manifold $(M, \Omega, \omega)$ and assume that the action admits an equivariant moment mapping $\psi$. Suppose $L$ is a maximal connected submanifold of $M$ with $T_{x} L=\zeta_{\mathfrak{g}}(x)^{\perp}$. Let $G_{L}$ be a subgroup of $G$ which preserves $L$ and has $\mathfrak{g}_{L}$ as Lie algebra, and assume that $G_{L}$ acts freely and properly on $L$. Then $P_{L}:=L / G_{L}$ admits a unique (up to conformal equivalence) l.c.s. structure $(\bar{\Omega}, \bar{\omega})$, such that $\left(L, i^{*} \Omega, i^{*} \omega\right) \sim\left(L, \pi^{*} \bar{\Omega}, \pi^{*} \bar{\omega}\right)$, where $i: L \hookrightarrow M$ denotes the inclusion and $\pi: L \rightarrow$ $P_{L}$ denotes the projection.

Proof. Since the action is free and proper $\pi: L \rightarrow P_{L}$ is a principle $G_{L}$-bundle. Let $\mathcal{U}$ be an open covering of $P_{L}$ and, for $U \in \mathcal{U}$, let $s_{U}: U \rightarrow L$ be local sections of $\pi$. Define $\bar{\Omega}_{U}:=s_{U}^{*} i^{*} \Omega$ and $\bar{\omega}_{U}:=s_{U}^{*} i^{*} \omega$. We claim that $\left(U, \bar{\Omega}_{U}, \bar{\omega}_{U}\right)$ is l.c.s. for every $U \in \mathcal{U}$. Obviously $d \bar{\omega}_{U}=0$ and $d^{\bar{\omega}_{U}} \bar{\Omega}_{U}=0$. To see that $\bar{\Omega}_{U}$ is non-degenerate, let $Y_{z} \in T_{z} P_{L}$ and suppose $0=\bar{\Omega}_{U}\left(Y_{z}, Z_{z}\right)=i^{*} \Omega\left(T_{z} s_{U} \cdot Y_{z}, T_{z} s_{U} \cdot Z_{z}\right)$ for all $Z_{z} \in T_{z} P_{L}$. Since $i_{\zeta_{X}} i^{*} \Omega=0$ for all $X \in \mathfrak{g}_{L}$, we conclude that $\Omega\left(T_{z} s_{U} \cdot Y_{z}, T_{s(z)} L\right)=0$. In other words $T_{z} s_{U} \cdot Y_{z} \in\left(T_{s_{U}(z)} L\right)^{\perp}=\zeta_{\mathfrak{g}}\left(s_{U}(z)\right)$ and thus $T_{z} s_{U} \cdot Y_{z}=0$, i.e. $Y_{z}=0$.

Next we will show that $\left.\left(i^{*} \Omega, i^{*} \omega\right)\right|_{\pi^{-1}(U)} \sim \pi^{*}\left(\bar{\Omega}_{U}, \bar{\omega}_{U}\right)$. So define $f: \pi^{-1}(U) \rightarrow$ $G$ by $l_{f(x)}(x):=s_{U}(\pi(x))$, and $a \in C^{\infty}\left(\pi^{-1}(U), \mathbb{R}^{+}\right)$by $a(x):=a_{f(x)}(x)$. We claim that $\pi^{*} \bar{\Omega}_{U}=\left.\frac{1}{a} i^{*} \Omega\right|_{\pi^{-1}(U)}$ and $\pi^{*} \bar{\omega}_{U}=\left.i^{*} \omega\right|_{\pi^{-1}(U)}+\frac{d a}{a}$. Indeed we have $T_{x}\left(s_{U} \pi\right) \cdot Z_{x}=T_{x} l_{f(x)} \cdot Z_{x}+V$ for a vertical $V \in T_{s_{U}(\pi(x))} L$. Since $i^{*} \Omega$ vanishes on vertical vectors we get

$$
\begin{aligned}
\left(\pi^{*} \bar{\Omega}_{U}\right)\left(Z_{x}, Z_{x}^{\prime}\right) & =\left(s_{U} \pi\right)^{*} i^{*} \Omega\left(Z_{x}, Z_{x}^{\prime}\right) \\
& =i^{*} \Omega\left(T_{x}\left(s_{U} \pi\right) \cdot Z_{x}, T_{x}\left(s_{U} \pi\right) \cdot Z_{x}^{\prime}\right) \\
& =i^{*} \Omega\left(T_{x} l_{f(x)} \cdot Z_{x}, T_{x} l_{f(x)} \cdot Z_{x}^{\prime}\right) \\
& =i^{*} l_{f(x)}^{*} \Omega\left(Z_{x}, Z_{x}^{\prime}\right) \\
& =\frac{1}{a(x)} i^{*} \Omega\left(Z_{x}, Z_{x}^{\prime}\right)
\end{aligned}
$$

that is $\pi^{*} \bar{\Omega}_{U}=\left.\frac{1}{a} i^{*} \Omega\right|_{\pi^{-1}(U)}$. Moreover we have $f(g x)=f(x) g^{-1}$ for $g \in G_{L}$, and therefore $a(g x)=\frac{1}{a_{g}(x)} a(x)$, where we used $a_{h g}(x)=a_{g}(x) a_{h}(g x)$. From this we obtain

$$
i_{\zeta_{Y}(x)} d a=\left.\frac{\partial}{\partial t}\right|_{0}\left(\frac{1}{a_{\exp (t Y)}(x)} a(x)\right)=-i^{*} \omega\left(\zeta_{Y}(x)\right) a(x),
$$

i.e. $\left(\pi^{*} \bar{\omega}_{U}-i^{*} \omega-\frac{d a}{a}\right)\left(\zeta_{Y}\right)=0$ for all $Y \in \mathfrak{g}_{L}$. Remains to check this equation on 'horizontal' vector fields. Since $f\left(s_{U}(z)\right)=e$ we have $s_{U}^{*} a=1$ and thus

$$
\left(l_{g} s_{U}\right)^{*}\left(i^{*} \omega+\frac{d a}{a}\right)=s_{U}^{*}\left(i^{*} \omega+\frac{d a_{g}}{a_{g}}+\frac{d l_{g}^{*} a}{l_{g}^{*} a}\right)=s_{U}^{*}\left(i^{*} \omega+\frac{d a}{a}\right)=\bar{\omega}_{U} .
$$

Since $\left(l_{g} s_{U}\right)^{*}\left(\pi^{*} \bar{\omega}_{U}\right)=\bar{\omega}_{U}$, we obtain $\pi^{*} \bar{\omega}_{U}=\left.i^{*} \omega\right|_{\pi^{-1}(U)}+\frac{d a}{a}$. 
If $V \in \mathcal{U}$ then we get

$$
\left.\left(\bar{\Omega}_{V}, \bar{\omega}_{V}\right)\right|_{U \cap V}=\left.\left.s_{V}^{*} i^{*}(\Omega, \omega)\right|_{U \cap V} \sim s_{V}^{*} \pi^{*}\left(\bar{\Omega}_{U}, \bar{\omega}_{U}\right)\right|_{U \cap V}=\left.\left(\bar{\Omega}_{U}, \bar{\omega}_{U}\right)\right|_{U \cap V}
$$

By the construction described in the first paragraph, we obtain a unique l.c.s. structure $(\bar{\Omega}, \bar{\omega})$ on $P_{L}$, such that $\left.(\bar{\Omega}, \bar{\omega})\right|_{U} \sim\left(\bar{\Omega}_{U}, \bar{\omega}_{U}\right)$ for all $U \in \mathcal{U}$. Moreover we have $\left.\left.\pi^{*}(\bar{\Omega}, \bar{\omega})\right|_{\pi^{-1}(U)} \sim i^{*}(\Omega, \omega)\right|_{\pi^{-1}(U)}$ for all $U \in \mathcal{U}$ and hence $\pi^{*}(\bar{\Omega}, \bar{\omega}) \sim i^{*}(\Omega, \omega)$. Uniqueness is also clear, for if $\left(\Omega^{\prime}, \omega^{\prime}\right)$ is any l.c.s. structure on $P_{L}$ with $\pi^{*}\left(\Omega^{\prime}, \omega^{\prime}\right) \sim$ $i^{*}(\Omega, \omega)$, then

$$
\left.\left(\Omega^{\prime}, \omega^{\prime}\right)\right|_{U}=s_{U}^{*} \pi^{*}\left(\Omega^{\prime}, \omega^{\prime}\right) \sim s_{U}^{*} i^{*}(\Omega, \omega)=\left(\bar{\Omega}_{U}, \bar{\omega}_{U}\right)
$$

and so $\left(\Omega^{\prime}, \omega^{\prime}\right) \sim(\bar{\Omega}, \bar{\omega})$.

Suppose $(M, \Omega, \omega) \sim\left(M, \Omega^{\prime}, \omega^{\prime}\right)$ with $\Omega^{\prime}=\frac{1}{a} \Omega$ and $\omega^{\prime}=\omega+\frac{d a}{a}$. Then $\operatorname{Diff}^{\infty}(M, \Omega, \omega)=\operatorname{Diff}^{\infty}\left(M, \Omega^{\prime}, \omega^{\prime}\right)$ and $\operatorname{Ham}(M, \Omega, \omega)=\operatorname{Ham}\left(M, \Omega^{\prime}, \omega^{\prime}\right)$. Moreover, $\psi$ is an equivariant moment mapping for $(\Omega, \omega)$ iff $\frac{1}{a} \psi$ is an equivariant moment mapping for $\left(\Omega^{\prime}, \omega^{\prime}\right)$. Since the orthogonal relation is the same for both l.c.s. structures a submanifold $L$ satisfies the conditions of Theorem 1 for $(\Omega, \omega)$ iff it does for $\left(\Omega^{\prime}, \omega^{\prime}\right)$. Moreover we have $\mathfrak{g}_{L}^{\prime}=\mathfrak{g}_{L}$. So the reduced spaces $\left(P_{L}, \Omega, \omega\right)$ and $\left(P_{L}^{\prime}, \Omega^{\prime}, \omega^{\prime}\right)$ are the same, up to conformal equivalence.

Let $a \in C^{\infty}(M, \mathbb{R})$ be such that $\omega=\frac{d a}{a}$, locally around $x_{0} \in L$. Then $L$ locally given as $\left\{x: \frac{1}{a} \psi(x)=\frac{1}{a} \psi\left(x_{0}\right)\right\}$.

If $(M, \Omega, \omega)$ is symplectic, i.e. $\omega=0$, then this construction yields of course ordinary symplectic reduction $([7],[3])$.

\section{EXAMPLES}

Let $G$ be a discrete group acting freely and properly on an l.c.s. manifold $(M, \Omega, \omega)$, such that $l_{g} \in \operatorname{Diff}^{\infty}(M, \Omega, \omega)$ for all $g \in G$. Then $\mathfrak{g}=0, \psi=0$ is an equivariant moment mapping, and the only possible choice for $L$ is $L=M$. If we choose $G_{L}=G$ then $P_{L}=M / G$ carries an l.c.s. structure. Notice that even if we start with a symplectic manifold, $M / G$ need not be conformally equivalent to a symplectic manifold. For example let $(M, \Omega, \omega)$ be an l.c.s. manifold, let $\pi: \tilde{M} \rightarrow M$ be a normal covering such that $\tilde{\omega}:=\pi^{*} \omega$ becomes exact, and set $\tilde{\Omega}:=\pi^{*} \Omega$. Then $(\tilde{M}, \tilde{\Omega}, \tilde{\omega})$ is conformally equivalent to a symplectic manifold and $G$, the group of deck transformations, satisfies $l_{g} \in \operatorname{Diff}^{\infty}(\tilde{M}, \tilde{\Omega}, \tilde{\omega})$ for all $g \in G$. Obviously $P_{L}=\tilde{M} / G=M$ and $(\bar{\Omega}, \bar{\omega}) \sim(\Omega, \omega)$. The smallest $\tilde{M}$ one can take is the covering corresponding to the kernel of the homomorphism $\pi_{1}(M) \rightarrow \mathbb{R}$, $\sigma \mapsto \int_{\sigma} \omega$.

Another example is connected with physical applications. In [9] the following l.c.s. reduction is applied in several dynamical systems. Let $(M, \Omega, \omega)$ be an l.c.s. manifold, and $f \in C^{\infty}(M, \mathbb{R})$ be a Hamiltonian function. One considers $F=\sharp d f$ and a smooth level set $M^{c}=\{x \in M: f(x)=c\}$, and one assumes that $f$ does not vanish on $M^{c}$. Then one obtains a reduced l.c.s. structure on $\hat{M}^{c}=M^{c} / F$. This structure enables to get a conformally symplectic transversal derivative cycle. Then some symmetries of the Lyapunov spectrum of this cocycle appear. For details, see $[9]$. 


\section{Hamiltonian System On The Reduced Space}

First we consider the general reduction and assume the notation of Proposition 1.

Proposition 4. Let $P_{L}$ be the reduced l.c.s. manifold by means of $(L, E)$ and let $u \in C^{\infty}(M, \mathbb{R})$ be a Hamiltonian such that $d^{\omega} u$ vanishes on $E$ and its flow $\mathrm{Fl}_{t}^{\sharp d} u$ preserves $L$ and the bundle $E$. Then there exists a unique $\bar{u} \in C^{\infty}\left(P_{L}, \mathbb{R}\right)$ such that $u$ extends $\bar{u} \circ \pi$ and $\pi_{*} \sharp d^{\omega} u=\sharp_{P_{L}} d^{\bar{\omega}} \bar{u}$. In particular, $\mathrm{Fl}_{t}^{\sharp d^{\omega} u}$ induces a flow of Hamiltonian automorphisms on $P_{L}$.

Proof. Since $\left.d^{\omega} u\right|_{E}=\left.d u\right|_{E}=0$, the $\bar{u}$ as above exists. For all $\bar{v} \in C^{\infty}\left(P_{L}, \mathbb{R}\right)$ and $v \in C^{\infty}(M, \mathbb{R})$ with $\left.v\right|_{L}=\bar{v} \circ \pi$ we then get

$$
\begin{aligned}
\left(\sharp_{P_{L}} d^{\bar{\omega}} \bar{u}\right)(\pi(x)) \bar{v} & =\{\bar{v}, \bar{u}\}_{P_{L}}(\pi(x))=\{v, u\}_{M}(x) \\
=\left(\sharp d^{\omega} u\right)(x) v & =\left(\sharp d^{\omega} u\right)(x)(\bar{v} \circ \pi)=\left(\pi_{*} \sharp d^{\omega} u\right)(x) \bar{v} .
\end{aligned}
$$

Here we use that, by assumption, $\sharp d^{\omega} u$ is tangent to $L$.

Now we consider the case of Lie group actions.

Lemma 1. Let $G$ and $H$ be two finite dimensional, connected Lie groups acting symplectically on an l.c.s. manifold $(M, \Omega, \omega)$ with moment mappings $\psi_{G}$ and $\psi_{H}$. Then

(1) $a_{g} l_{g}^{*} \psi_{H}=\psi_{H}$, for all $g \in G$,

(2) $a_{g} l_{g}^{*} \hat{\psi}_{H}(Y)=\hat{\psi}_{H}(Y)$, for all $Y \in \mathfrak{h}, g \in G$,

(3) $T \psi_{H} \cdot \zeta_{X}^{G}+\omega\left(\zeta_{X}^{G}\right) \psi_{H}=0$, for all $X \in \mathfrak{g}$,

(4) $L_{\zeta_{X}^{G}}^{\omega} \hat{\psi}_{H}(Y)=0$, for all $Y \in \mathfrak{h}, X \in \mathfrak{g}$,

(5) $\zeta_{\mathfrak{h}}^{H}(x) \subseteq \zeta_{\mathfrak{g}}^{G}(x)^{\perp}$, for all $x \in M$ and

(6) $\left\{\hat{\psi}_{G}(X), \hat{\psi}_{H}(Y)\right\}=0$, for all $X \in \mathfrak{g}, Y \in \mathfrak{h}$

are equivalent. Moreover each statement is equivalent to the corresponding statement with the rôles of $G$ and $H$ exchanged. If these equivalent conditions are satisfied, then $\left[\zeta_{X}^{G}, \zeta_{Y}^{H}\right]=0$ for all $X \in \mathfrak{g}, Y \in \mathfrak{h}$ and the actions of $G$ and $H$ commute.

Proof. From $\Omega\left(\zeta_{X}^{G}, \zeta_{Y}^{H}\right)=-L_{\zeta_{X}^{G}}^{\omega} \hat{\psi}_{H}(Y)=\left\{\hat{\psi}_{G}(X), \hat{\psi}_{H}(Y)\right\}$ we obtain the equivalence of (4), (5) and (6). Since and $L_{Z}^{\omega} \hat{\psi}(Y)=\langle T \psi \cdot Z+\omega(Z) \psi, Y\rangle$ these are equivalent to (3). (1) and (2) are the infinitesimal versions of (3) and (4). The equivalence to the statements with the rôles of $G$ and $H$ exchanged is obvious from the symmetry of (5) or (6). From (6) we also get $\left[\zeta_{X}^{G}, \zeta_{Y}^{H}\right]=-\sharp d^{\omega}\left\{\hat{\psi}_{G}(X), \hat{\psi}_{H}(Y)\right\}=0$, i.e. the actions of $G$ and $H$ commute.

Assume that the equivalent conditions of Lemma 1 are satisfied and suppose $L$ satisfies the assumptions of Theorem 1 . Then $\zeta_{Y}^{H}$ is tangential to $L$ and thus at least $H_{\circ}$, the id component of $H$, leaves $L$ invariant.

Theorem 2. Let $(M, \Omega, \omega), G, L$ and $G_{L}$ satisfy the conditions of Theorem 1. Moreover let $H$ be another finite dimensional Lie group, acting symplectically on $(M, \Omega, \omega)$ with equivariant moment mapping $\psi_{H}$, such that $a_{g} l_{g}^{*} \psi_{H}=\psi_{H}$ for all $g \in G$. Finally assume that the actions of $G$ and $H$ commute and suppose that 
$H$ leaves $L$ invariant. Then the $H$-action descends to a symplectic action on $P_{L}$, which has an equivariant moment mapping naturally induced from $\psi_{H}$.

Proof. Since the action of $G$ and $H$ commute the action of $H$ descends to an action $\bar{l}$ on $P_{L}$. Moreover we have

$$
\pi^{*} \bar{l}_{h}^{*}(\bar{\Omega}, \bar{\omega})=l_{h}^{*} \pi^{*}(\bar{\Omega}, \bar{\omega}) \sim l_{h}^{*} i^{*}(\Omega, \omega)=i^{*} l_{h}^{*}(\Omega, \omega) \sim i^{*}(\Omega, \omega),
$$

and thus the uniqueness part of Theorem 1 yields $\bar{l}_{h}^{*}(\bar{\Omega}, \bar{\omega}) \sim(\bar{\Omega}, \bar{\omega})$, i.e. $H$ acts symplectically on $P_{L}$. Now let $a \in C^{\infty}\left(L, \mathbb{R}^{+}\right)$be such that $\pi^{*} \bar{\Omega}=\frac{1}{a} i^{*} \Omega$ and $\pi^{*} \bar{\omega}=i^{*} \omega+\frac{d a}{a}$. From $a_{g} l_{g}^{*} \psi_{H}=\psi_{H}$ and $a_{g} l_{g}^{*} a=a$ for all $g \in G_{L}$, we obtain $l_{g}^{*}\left(\frac{1}{a} \psi_{H}\right)=\frac{1}{a} \psi_{H}$ on $L$, and thus $\frac{1}{a} \psi_{H}$ descends to $\bar{\psi}_{H} \in C^{\infty}\left(P_{L}, \mathfrak{h}^{*}\right)$. Moreover we have

$$
\pi^{*}\left(\bar{a}_{h} \bar{l}_{h}^{*} \bar{\psi}_{H}\right)=\left(\pi^{*} \bar{a}_{h}\right) l_{h}^{*}\left(\frac{1}{a} \psi_{H}\right)=\operatorname{Ad}_{h^{-1}}^{*}\left(\frac{1}{a} \psi_{H}\right)=\pi^{*}\left(\operatorname{Ad}_{h^{-1}}^{*} \bar{\psi}_{H}\right)
$$

and hence $\bar{\psi}_{H}$ is equivariant. Since we have

$$
\pi^{*} d^{\bar{\omega}} \hat{\bar{\psi}}_{H}(Y)=d^{\pi^{*} \bar{\omega}}\left(\frac{1}{a} \hat{\psi}(Y)\right)=i_{\zeta_{Y}^{H}} \pi^{*} \bar{\Omega}=\pi^{*} i_{\bar{\zeta}_{Y}^{H}} \bar{\Omega},
$$

$\psi_{H}$ is a moment mapping.

Suppose $G$ is connected and let $L$ satisfy the conditions of Theorem 1 . Let $f$ be a Hamiltonian function, $H=\mathbb{R}$ acting via the flow of $\sharp d^{\omega} f$. Then $f$ is an equivariant moment mapping. Suppose $a_{g} l_{g}^{*} f=f$ for all $g \in G$, or equivalently $L_{\zeta_{X}^{G}}^{\omega} f=0$ for all $X \in \mathfrak{g}$. Then by Lemma 1 all assumptions of Theorem 2 are satisfied, and one obtains an induced Hamiltonian system on $P_{L}$.

\section{REFERENCES}

1. J.M.Arms, M.J.Gotay and G.Jennings, Geometric and algebraic reduction for singular momentum maps, Adv. Math. 79 (1990), 43-103.

2. F.Guedira and A.Lichnerowicz, Géométrie des algébres de Lie locales de Kirillov, J. Math. Pures Appl. 63 (1984), 407-484.

3. V.Guillemin and S.Sternbrg, Cambridge Univ. Press (1984).

4. S.Haller and T.Rybicki, On the Group of Diffeomorphisms Preserving a Locally Conformal Symplectic Structure, Ann. Global Anal. and Geom. 17 (1999), 475-502.

5. H.C.Lee, A kind of even dimensional differential geometry and its application to exterior calculus, Amer. J. Math. 65 (1943), 433-438.

6. J.E.Marsden, T.Ratiu, Reduction of Poisson manifolds, Lett. Math. Phys. 11 (1986), 161-169.

7. J.Marsden and A.Weinstein, Reduction of symplectic manifolds with symmetry, Rep. Mathematical Phys. 5 (1974), 121-130.

8. I.Vaisman, Locally conformal symplectic manifolds, Internat. J. Math. Math. Sci. 8 (1985), 521-536.

9. M.P.Wojtkowski, C.Liverani, Conformally Symplectic Dynamics and Symmetry of the Lyapunov Spectrum, Comm. Math. Phys. 194 (1998), 47-60.

Stefan Haller, Institute of Mathematics, University of Vienna, Strudlhofg. 4, A-1090 Vienna, Austria

Current address: Stefan Haller, Departement of Mathematics, Ohio State University, 231 West 18th Avenue, Columbus, Ohio 43210, USA

E-mail address: stefan@mat.univie.ac.at

Tomasz Rybicki, Dept. of Applied Mathematics at AGH, al. Mickiewicza 30, 30059 Cracow, Poland

E-mail address: tomasz@uci.agh.edu.pl 